2319. Molecular Epidemiology of Staphyloccoccus aureus Isolated From Korean Children

Chan Jae Lee, $\mathrm{MD}^{1}$; Hyeon Seung Lee, $\mathrm{MD}^{2}$; Hyunju Lee, MD, $\mathrm{PhD}^{3,4}$; Mi Seon Han, $\mathrm{MD}^{5}$; Ki Wook Yun, $\mathrm{MD}, \mathrm{PhD}^{4,5}$; Eun Hwa Choi, $\mathrm{MD}, \mathrm{PhD}^{4,5}$ and Hoan Jong Lee, $\mathrm{MD}, \mathrm{PhD}^{4,5}$; ${ }^{1}$ Pediatrics, Seoul National University Bundang Hospital, Seongnam, Korea, Republic of (South), ${ }^{2}$ Pediatrics, Seoul National University College of Medicine, Seoul, Korea, Republic of (South), ${ }^{3}$ Department of Pediatrics, Seoul National University Bundang Hospital, Seongnam, Korea, Republic of (South), ${ }^{4}$ Department of Pediatrics, Seoul National University College of Medicine, Seoul, Korea, Republic of (South), ${ }^{5}$ Department of Pediatrics, Seoul National University Children's Hospital, Seoul, Korea, Republic of (South)

Session: 247. Pediatric Bacterial Infections

Saturday, October 6, 2018: 12:30 PM

Background. Major community acquired-methicillin-resistant Staphylococcus aureus (CA-MRSA) clones have been reported worldwide including ST1 in Asia, Europe, United States, ST8 in Europe and United States and ST30 in Australia, Europe and South America. Panton-Valentine leukocidin (PVL) positive ST30-SCCmec IV has been reported as an important CA-MRSA in Singapore, Japan and Latin America, however reports in Korean children are limited. Therefore we investigated the antimicrobial resistance and molecular characteristics of $S$. aureus among children in Korea.

Methods. S. aureus previously isolated from children at Seoul National University Bundang Hospital (2010-2016) were analyzed for multilocus sequence type, SCCmec typing, and PCR of $P V L, q a c \mathrm{~A} / \mathrm{B}, s m r$ and mupA genes. Electronic medical records were reviewed for clinical data and antibiotic susceptibility results.

Results. A total of 79 isolates from skin and soft-tissue infection (SSTI) $(N=41,51.9 \%)$, bone and joint infection $(N=26,32.9 \%)$ and staphylococcal scalded skin syndrome (SSSS) $(N=12,15.2 \%)$ were included. Among these, $32(40.5 \%)$ were MRSA. Among children with underlying diseases (20.3\%, 16/79), 56.3\% (9/16) were MRSA. After excluding these cases, among children $\leq 1$ month of age, $84.6 \%(11 / 13)$ were MRSA, whereas in children $\geq 2$ months of age, $95.2 \%$ (20/21) of SSTI, and $90.0 \%$ $(18 / 20)$ of bone and joint infection were MSSA. All SSSS cases were MRSA. Among MSSA strains, ST30 $(N=28,59.6 \%)$ was the predominant clone and among ST30, $96.6 \%(28 / 29)$ were MSSA. MRSA strains included ST72-SCCmec IV $(N=15,46.9 \%)$ ST89-SCCmec IV $(N=10,31.3 \%)$, ST 5-SCCmec II $(N=3,9.4 \%)$ and ST1-SCCmec IV $(N=3,9.4 \%)$. ST30 was the most common clone in SSTI and bone and joint infection whereas ST89-SCCmec IV was most common in SSSS. PVL was detected in 3 strains $(3.8 \%$, ST30-SCCmec IV $N=1$, MSSA ST30 $N=2)$ and qac A/B in 3 strains $(\mathrm{MRSA}=3)$, smr in 3 strains (MSSA $=1, \mathrm{MRSA}=2)$ and $m u p \mathrm{~A}$ in 7 strains $(\mathrm{MRSA}=5$, MSSA = 2)

Conclusion. The molecular epidemiology of S. aureus in Korean children differed from other countries. Among children with SSTI and bone and joint infection, ST30 was the predominant strain, and the majority was MSSA. Among MRSA isolates, ST72-SCCmec type IV was the most common clone in SSTI and bone and joint infection, and ST89-SCCmec type IV in SSSS.

Disclosures. H. Lee, National Research Foundation of Korea: Grant Investigator, Grant recipient

2320. Decolonization of the Oropharynx, an Important and Neglected Reservoir of Staphylococcus aureus Colonization

Evelyn A. Flores, $\mathrm{BS}^{1}$; Bryn Launer, $\mathrm{BA}^{2}$; Kelly Young, $\mathrm{MD}^{3}$; Gregory K. Tchakalian, $\mathrm{BA}^{1}$; Michael Bolaris, $\mathrm{MD}^{4}$; Pooja Modi, $\mathrm{MD}^{1}$; Kyle Ramsay, BS ${ }^{4}$; Alex Varasteh, $\mathrm{BA}^{5}$ and Loren G. Miller, MD, $\mathrm{MPH}^{6} ;{ }^{1}$ Los Angeles Biomedical Research Institute, Torrance, California, ${ }^{2}$ University of Colorado Denver School of Medicine, Aurora, Colorado, ${ }^{3}$ Harbor-UCLA Medical Center, Torrance, California, ${ }^{4}$ Infectious Disease Clinical Outcomes Research Unit, Division of Infectious Disease, Los Angeles Biomedical Research Institute at Harbor-University of California Los Angeles Medical Center, Torrance, California, ${ }^{5}$ Infectious Disease Clinical Outcomes Research (ID-CORE), LA Biomed at Harbor-UCLA Medical Center, Torrance, California, ${ }^{6}$ Medicine, David Geffen School of Medicine at University of California, Los Angeles., Los Angeles, California

Session: 247. Pediatric Bacterial Infections

Saturday, October 6, 2018: 12:30 PM

Background. Community-associated S. aureus skin and soft-tissue infections are common and recur in 20 to $>50 \%$ of cases. Decolonization trials have been disappointing for unclear reasons, but may be related to untreated reservoirs. Given recent data that oropharyngeal (OP) S. aureus colonization is common with a prevalence comparable to nasal colonization, we performed a double-blind, placebo controlled trial of the efficacy of oral chlorhexidine gluconate (CHG) for OP S. aureus colonization.

Methods. We enrolled healthy outpatient children from ages 5 to 17 who were tested for OP S. aureus colonization. Colonized subjects were randomized to $0.12 \%$ $\mathrm{CHG}$ or placebo gargle twice daily $\times 7$ days. Primary endpoint was OP colonization at the End of Therapy (EOT) visit using an intention to treat (ITT) model. We also measured OP colonization at 28 days and nasal S. aureus colonization at all study visits.

Results. Among 189 consented subjects, 120 (63\%) had OP colonization; 81/120 $(66 \%)$ were randomized and 67 were analyzable (CHG: $N=33$; Placebo: $N=34$ ). Fourteen subjects were not analyzable due to product error or loss to follow-up prior to study drug receipt (figure). In the ITT analysis, EOT OP S. aureus colonization was $45 \%(15 / 33)$ in the $\mathrm{CHG}$ group and $79 \%(27 / 34)$ in the placebo group $(P=0.004)$. In the as treated analysis, OP colonization was $40 \%(11 / 29)$ and $77 \%(23 / 30)$ in the CHG group and placebo groups $(P=0.003)$. At Day 28 in the ITT model, OP colonization was $61 \%(20 / 33)$ vs. $85 \%(29 / 34)$ in the CHG and placebo groups $(P=0.03)$. At EOT nasal colonization in those without OP colonization was $11 / 25(44 \%)$ vs. $15 / 34(44 \%)$ in those still OP colonized. At Day 28, nasal colonization was $0 / 18(0 \%)$ in those with out OP colonization vs. 19/38 (50\%) in those with OP colonization.

Conclusion. One week of $0.12 \%$ oral CHG gargle was more effective than the placebo at eradicating $S$. aureus OP colonization in $S$. aureus colonized children. Significant differences persisted at Day 28. Persistent OP colonization at Day 28 was associated with nasal $S$. aureus colonization, suggesting that nasal colonization may contribute to persistence and relapse of OP S. aureus colonization. Our findings support decolonization trials that include OP $S$. aureus decolonization as part of a more aggressive $S$. aureus decolonization strategy.

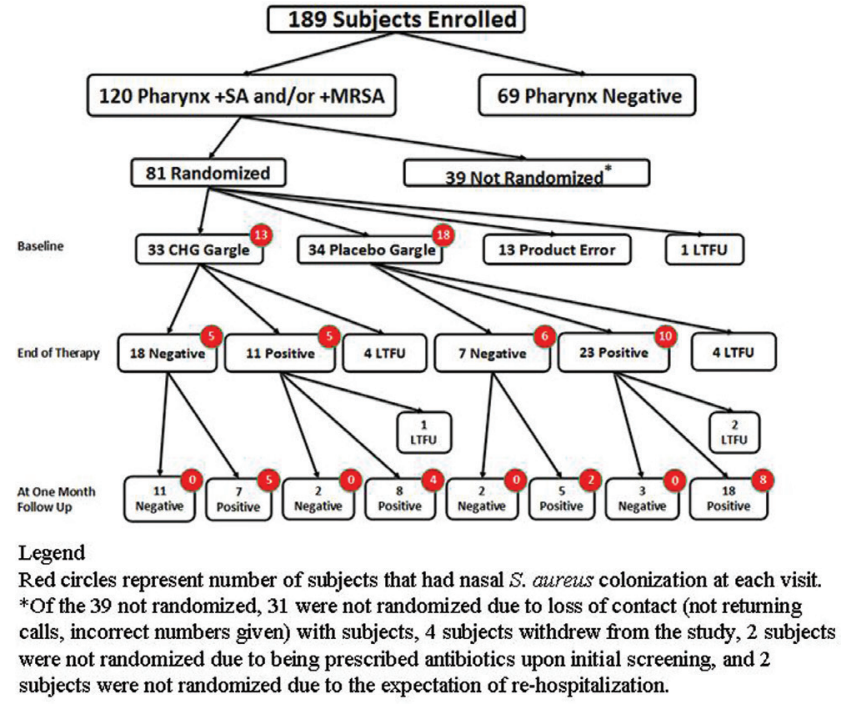

Disclosures. All authors: No reported disclosures.

2321. Epidemiology of Staphylococcus aureus Infections in Patients Admitted to Freestanding Pediatric Hospitals, 2009-2016

Alicen B. Spaulding, $\mathrm{PhD}, \mathrm{MPH}^{1}$; Cary Thurm, $\mathrm{PhD}^{2}$; Joshua Courter, PharmD 3 ; Ritu Banerjee, $\mathrm{MD}, \mathrm{PhD}^{4}$; Jeffrey S. Gerber, $\mathrm{MD}, \mathrm{PhD}^{5}$; Jason Newland, $\mathrm{MD}$, MEd, FPIDS ${ }^{6}$; Sarah Parker, $\mathrm{MD}^{7}$; Thomas Brogan, $\mathrm{MD}^{8}$; Matthew Kronman, MD, MSCE; Samir Shah, $\mathrm{MD}^{10}$; Michael Smith, MD, MSCE ${ }^{11}$; Sameer Patel, MD, MPH ${ }^{12}$;

Brian R. Lee, $\mathrm{MPH}, \mathrm{PhD}^{13}$ and Adam L. Hersh, MD, $\mathrm{PhD}^{14} ;{ }^{1}$ Children's Minnesota Research Institute, Minneapolis, Minnesota, ${ }^{2}$ Children's Hospital Association, Overland Park, Kansas, ${ }^{3}$ Division of Pharmacy, Cincinnati Children's Hospital Medical Center, Cincinnati, Ohio, ${ }^{4}$ Division of Pediatric Infectious Diseases, Vanderbilt University, Nashville, Tennessee, ${ }^{5}$ Department of Pediatrics, Division of Infectious Diseases, The Children's Hospital of Philadelphia, Philadelphia, Pennsylvania, ${ }^{6}$ Washington University S, Kansas City, Missouri, ${ }^{7}$ Pediatrics, Children's Hospital Colorado/University of Colorado School of Medicine, Aurora, Colorado, ${ }^{8}$ Seattle Childrens, Seattle, Washington, ${ }^{9}$ Pediatrics, University of Washington, Seattle, Washington, ${ }^{10}$ Division of Hospital Medicine, Cincinnati Children's Hospital Medical Center, Cincinnati, Ohio, ${ }^{11}$ Pediatric Infectious Diseases, Duke University, Durham, North Carolina, ${ }^{12}$ Pediatric Infectious Diseases, Ann \& Robert H. Lurie Children's Hospital of Chicago, Chicago, Illinois, ${ }^{13}$ Pediatrics, Hasbro Children's Hospital, Brown University, Providence, Rhode Island, ${ }^{14}$ University of Utah School of Medicine, Salt Lake City, Utah

Session: 247. Pediatric Bacterial Infections

Saturday, October 6, 2018: 12:30 PM

Background. S. aureus causes a substantial number of pediatric infections in the United States each year, with potential for severe complications including death. Recent data suggest $S$. aureus infections are declining in adults, but a nationally representative and contemporary characterization of trends in pediatric $S$. aureus infections is lacking. Our objective was to describe recent pediatric hospitalization trends for S. aureus and associated antibiotic prescribing patterns.

Methods. We conducted a retrospective cohort study using Pediatric Health Information Systems data from 39 tertiary care freestanding children's hospitals in the United States. All inpatient encounters for patients ages $<18$ hospitalized between $1 / 1 / 2009-12 / 31 / 2016$ at a continuously reporting hospital were included. Analysis was limited to patients with $S$. aureus infection, defined as: 1) having $\geq 1$ ICD discharge code for methicillin-resistant (MRSA) or methicillin-susceptible (MSSA) S. aureus, and 2) $\geq 1$ anti-staphylococcal antibiotic received. Analysis for rates were per 1,000 hospital admissions, antibiotic days of therapy (DOT) per 1,000 patient-days, and trends were analyzed using Cochran-Armitage tests; significance was set at $P<0.05$.

Results. From 2009-2016 we identified 116,152 S. aureus hospitalizations. Patients had median age 3 (interquartile range: $0-11$ years); $53.7 \%$ were male, $52.5 \%$ non-Hispanic white, and 18.8\% non-Hispanic African American. From 2009 to 2016, S. aureus hospitalizations declined $36 \%$ from 26.3 to 16.8 infections per 1,000 
admissions $(P<0.001)$ (figure). MRSA infections declined 52\% (14.4 in 2009 to 6.9 infections per 1,000 admissions in 2016, $P<0.001$ ) while MSSA infections declined $17 \%$ (11.9 to 9.9 infections per 1,000 admissions, $P<0.001$ ). DOT for anti-MRSA antibiotics declined from 38.0 to 24.5 per 1,000 patient-days.

Conclusion. Rates of pediatric hospitalization with S. aureus infection declined substantially over time. This was largely driven by decreased rates of MRSA hospitalizations, and we observed a corresponding decline in anti-MRSA antibiotic use. Further research is needed to better understand factors driving epidemiologic changes.

Figure. S. aureus hospitalization rate per 1,000 hospital admissions in 39 PHIS hospitals with continuous reporting, 2009-2016.

All S. aureus

infections

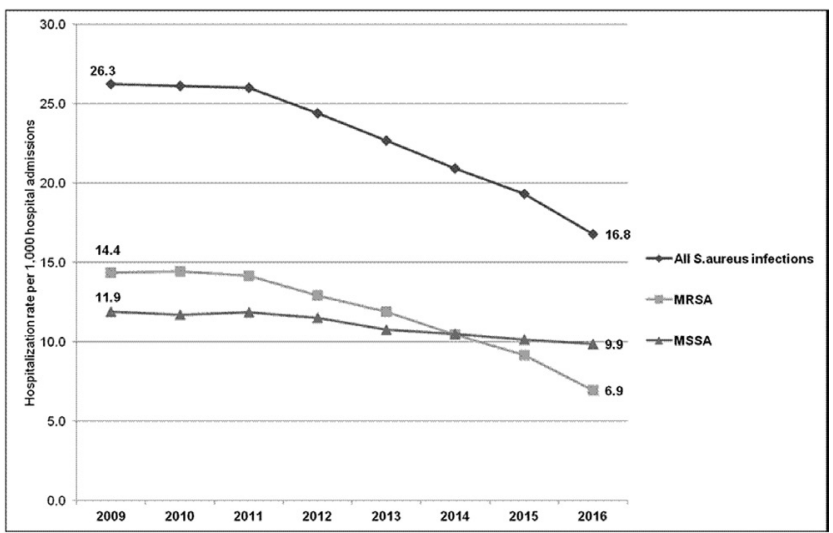

Disclosures. R. Banerjee, Accelerate Diagnostics, Biomerieux, BioFire: Gran Investigator, Research grant and Research support. S. Patel, Merck: Grant Investigator, Grant recipient and Research grant. Pfizer: Grant Investigator, Research grant.

2322. Reduced Vancomycin Susceptibility Among Pediatric Staphylococcus aureus Bloodstream Infections

Jessica Ericson, $\mathrm{MD}, \mathrm{MPH}^{1}$ and Ethan Canty, $\mathrm{BA}^{2} ;{ }^{1}$ Pediatrics, Penn State College of Medicine, Hershey, Pennsylvania, ${ }^{2}$ Penn State College of Medicine, Hershey, Pennsylvania

Session: 247. Pediatric Bacterial Infections

Saturday, October 6, 2018: 12:30 PM

Background. Reduced vancomycin susceptibility (RVS) is considered to be present when the minimum inhibitory concentration (MIC) is equal to $2 \mu \mathrm{g} / \mathrm{mL}$. RVS Staphylococcus aureus (SA) bloodstream infections (BSI) have been associated with worse outcomes than non-RVS BSI in adults but not been well studied in children.

Methods. We reviewed the electronic medical records of infants and children admitted to Penn State Children's Hospital with $\geq 1$ blood culture positive for SA from 2005 to 2015. We abstracted demographic information, potential risk factors, laboratory results and clinical outcomes. We defined RVS as a vancomycin MIC $=2 \mu \mathrm{g} / \mathrm{mL}$ as determined by the clinical microbiology laboratory at the time of the infection. We used Chi square and Wilcoxon rank-sum tests to compare patient factors for RVS and non-RVS infections. Using a logistic regression adjusted for year and the presence of an infection-related complication, we calculated the odds of treatment failure for children with RVS and non-RVS BSI. For children with a central line in place at the time of the first positive culture, we also calculated the odds of treatment failure adjusted for year, presence of a complication and line removal. We defined treatment failure as death within 30 days of the first positive culture, recurrence of SA BSI within 30 days or a duration of bacteremia $>3$ days.

Results. Of the 216 identified pediatric SA BSI, 139 (64\%) had RVS: RVS was present in $63 \%$ of MSSA BSI and $65 \%$ of MRSA BSI, $P=0.835$. There was no difference in age, sex, and racial distributions among children with RVS vs. non-RVS BSI. Similarly, hospitalization in the prior year, surgery within the prior 30 days, the presence of an underlying comorbidity or use of immunosuppressing medications were not more common for RVS vs. non-RVS BSI. RVS was not associated with an increased risk of treatment failure overall, odds ratio $(\mathrm{OR})=1.34$ (95\% confidence interval: $0.71,2.55$ ), but did increase the odds of treatment failure if an indwelling central venous catheter was present and not removed, $\mathrm{OR}=3.14(1.16,8.54)$.

Conclusion. RVS is common among pediatric SA BSI. For central line associated SA BSI, RVS was associated with increased odds of treatment failure compared with non-RVS infections if the line was retained.

Disclosures. All authors: No reported disclosures.

\section{Unexpected Pediatric Presentation Patterns of Toxic Shock Syndrome} AlizaSpaeth-Cook, BA, BS ${ }^{1}$; Ross Comisford, B.S. ${ }^{1}$ and Guliz Erdem, MD $^{2} ;{ }^{1}$ Infectious Disease, Nationwide Children's Hospital, Columbus, Ohio, ${ }^{2}$ Pediatrics, Nationwide Children's Hospital and the Ohio State University School of Medicine, Columbus, Ohio

Session: 247. Pediatric Bacterial Infections

Saturday, October 6, 2018: 12:30 PM
Background. A subcategory of severe septic shock, toxic shock syndrome (TSS) represents up to $20 \%$ of pediatric septic shock in the United States. Diagnostic criteria for streptococcal TSS (STSS) and non-streptococcal TSS (NSTSS) were first published by the CDC in the early 1990s, with updates, respectively, in 2010 and 2011 .

Methods. The Nationwide Children's Hospital electronic medical record was queried for inpatient hospitalizations with ICD-9/10 codes of interest between 1/1/2010 and $8 / 31 / 2017$. The query returned 579 hospitalizations which were assessed for adher ence to STSS and NSTSS criteria published by the CDC. 61 cases of TSS were identified: 27 STSS, 32 NSTSS. The prevalence of organ system involvement was quantified, and organ system involvement unanticipated by CDC criteria was examined for prevalence, quality and chronology.

Results. TSS patients were predominately female (62\%) with an average age of 12 . The most common presentation of TSS was with hypotension $(93 \%)$, fever $(82 \%)$ and rash $(72 \%)$. Findings unanticipated by CDC criteria include: pyuria in STSS (41\%), pulmonary involvement in NSTSS (66\%) and coagulation abnormalities in NSTSS (92\%). Pyuria in STSS was commonly accompanied by protein (73\%) and leukocyte esterase (55\%) on urinalysis. Pyuria also commonly presented with hematuria (45\%). Radiographic evidence of pulmonary involvement in NSTSS was typically described as bilateral/diffuse airspace disease, presenting simultaneously with pulmonary edema and pleural effusions. Abnormalities in PT/PTT associated with NSTSS were commonly found within the first few hours of admission and began normalizing by the next day; d-dimer assays were abnormal in the six instances in which they were assessed.

Conclusion. This study suggests that early signs and symptoms of pediatric TSS may exist beyond those described by existing guidelines. The organ systems found to be involved in this review are often found early in the clinical course and can be assessed by noninvasive methods. Contextualization of these findings within the narrative of TSS might help clinicians better detect and diagnose a disease associated with significant patient morbidity and mortality. They may also aid in understanding the results of toxic shock surveillance efforts.

Disclosures. All authors: No reported disclosures.

2324. Long-Term Health Outcomes of Children Evaluated for Unexplained Fevers in a Pediatric Infectious Diseases Clinic

Kathryn Weakley, $\mathrm{MD}^{1}$; Gary S. Marshall, $\mathrm{MD}^{2}$ and Victoria Statler, MD, MSc ${ }^{2}$ ${ }^{1}$ Pediatrics, University of Louisville, Louisville, Kentucky, ${ }^{2}$ Pediatrics, University of Louisville School of Medicine, Louisville, Kentucky

Session: 247. Pediatric Bacterial Infections

Saturday, October 6, 2018: 12:30 PM

Background. Unexplained fever is a common reason for outpatient referral to pediatric infectious diseases (PID) subspecialists. A previous study at our center concluded that most children referred to PID for prolonged or recurrent unexplained fever have self-limited illnesses and receive no specific diagnosis. Studies looking at longterm outcomes of such patients have not been published.

Methods. The study cohort consisted of 156 patients seen in the PID clinic for unexplained fever from 2008 through 2012 who were not given a definitive diagnosis or were thought to have sequential self-limited illnesses, plus 20 patients seen during that time who were diagnosed with periodic fever, aphthous stomatitis, pharyngitis, and adenitis (PFAPA) syndrome. A scripted telephone interview with consenting parents or guardians who could be reached was conducted in 2018

Results. Attempts were made to contact all 176 families; to date, 100 interviews have been completed. Thirteen of the children initially had prolonged, 45 recurrent, and 25 periodic fever; 17 had PFAPA. The mean follow-up period was 8 years. Only 2 patients developed new diagnoses in the interval since their initial PID visit. One who was thought to have PFAPA developed genital ulcers and was diagnosed with Behcet's disease 4 years after the PID visit. Another who was thought to have self-limited, prolonged fever was eventually diagnosed with juvenile idiopathic arthritis. None of the remaining 98 children developed serious diagnoses. However, 14 of these children reportedly have continued fevers; 9 of the children suffer from anxiety, and 4 of the remaining 5 report good general health

Conclusion. Most children seen in PID clinic for unexplained fever who were not given a specific diagnosis remained well after their initial visit. Two were diagnosed with autoinflammatory diseases after the appearance of telltale signs and symptoms, and none were diagnosed with immunodeficiency or cancer. The children who reportedly continue having fevers but are otherwise healthy warrant further study, with particular attention to their families' health and illness beliefs.

Disclosures. All authors: No reported disclosures.

2325. Bloodstream Infections in Hospitalized Children in the United States: Incidence, Pathogens, and Regional Differences

Alicen B. Spaulding, $\mathrm{PhD}, \mathrm{MPH}^{1}$; Dave Watson, $\mathrm{PhD}^{1}$; Jill Dreyfus, $\mathrm{PhD}, \mathrm{MPH}^{2}$; Ellen Bendel-Stenzel, $\mathrm{MD}^{3}$; Phillip Heaton, $\mathrm{PhD}^{4}$; Steve Grapentine, PharmD ${ }^{5}$ and Anupam Kharbanda, MD, MS ; ${ }^{1}$ Children's Minnesota Research Institute, Minneapolis, Minnesota, ${ }^{2}$ Premier Applied Sciences, Premier, Inc., Stillwater

Minnesota, ${ }^{3}$ Children's Minnesota Research Institute, Minnesota Neonatal Physicians, Minneapolis, Minnesota, ${ }^{4}$ Children's Minnesota, Minneapolis, Minnesota, ${ }^{5}$ Pharmacy, Children's Minnesota, Minneapolis, Minnesota, ${ }^{6}$ Pediatric Emergency Medicine, Children's Minnesota, Minneapolis, Minnesota 